\title{
Retrospective analysis of renal histology in asymptomatic patients with probable chronic kidney disease of unknown aetiology in Sri Lanka
}

\author{
S Wijetunge ${ }^{1}$, N V I Ratnatunga ${ }^{1}$, D T D J Abeysekera ${ }^{3}$, A W M Wazil ${ }^{3}$, M Selvarajah $^{4}$, C N Ratnatunga ${ }^{2}$ \\ (Index words: chronic kidney disease, renal failure hisopathology, Sri Lanka)
}

\begin{abstract}
Objectives To study the early pathological changes in renal lesions of asymptomatic patients with kidney diseases, with no definite aetiology living in regions endemic for chronic kidney disease of unknown etiology (CKDUe).
\end{abstract}

Design Retrospective study.

Setting Regions endemic for CKDUe in and around the North Central Province of Sri Lanka.

Study population Two hundred and eleven asymptomatic patients living in endemic regions detected with renal disease by screening for proteinuria using the dipstick method. Those with long standing hypertension, diabetes mellitus, histological diagnosis of primary glomerular diseases, immunocomplex mediated diseases or renal lesions secondary to systemic diseases were excluded.

Measurements Renal lesions were divided into seven histological categories depending on the pathological changes: Category 0: no detectable changes. Category 1: Interstitial fibrosis \pm mild interstitial inflammation \pm tubular atrophy; no glomerulosclerosis. Category 2: Interstitial fibrosis \pm mild interstitial inflammation \pm tubular atrophy; glomerulosclerosis. Category 3: Moderate or severe interstitial fibrosis, interstitial inflammation and tubular atrophy \pm glomerulosclerosis; Category 4: Interstitial inflammation \pm tubular atrophy \pm glomerulosclerosis; no interstitial fibrosis. Category 5: The prominent change is interstitial inflammation with tubulitis. Category 6: Severely scarred kidney. Histological categories were compared with calculated glomerular filtration rates and age of the patients.

Results Number of cases in histological categories 0 to 6 were: 7 (3.3\%), 71 (33.6\%), 53 (25.1\%), 63 (29.9\%), 0, $2(0.9 \%)$ and $15(7.1 \%)$ respectively. The mean glomerular filtration rate was $>90 \mathrm{ml} / \mathrm{min}$ in patients in category 0 and 1 and declined progressively in categories 2 and 3 . Apart from category 0 , all had interstitial fibrosis and in category 1, 62 (87.3\%) had interstitial fibrosis without inflammation. Severity of interstitial inflammation increased from category 1 to 3 .

Conclusions The early disease among asymptomatic patients is characterized by interstitial fibrosis without significant interstitial inflammation and glomerular sclerosis with preserved glomerular function. Although the role of interstitial inflammation in the initiation of the disease is not clear, it appears to have a role in the progression of the disease.

Ceylon Medical Journal 2013; 58: 142-7

\section{Introduction}

Chronic kidney disease of unknown aetiology (CKDU) is endemic among several rural farming communities in and around the North Central Province (NCP) of Sri Lanka, namely, Medawachchiya, Padaviya, Siripura, Horowpathana, Medirigiriya, Polonnaruwa, Nikawewa, Dehiattakandiya and Girandurukotte [1-4]. A WHO report has indicated that about $15 \%$ of the people, in the age group 15-70 years, in these regions are affected by CKDU [5]. Repeatedly elevated albumin - creatinine ratio of $\geq 30 \mathrm{mg} / \mathrm{g}$ was used as the diagnostic criterion of CKD in that study. Compared to the other regions in the country, in these endemic regions, most patients have CKDU [2]. The disease typically has an insidious onset with gradual progression towards end stage renal disease.

Although studies have been carried out to determine the aetiology of this disease a definite aetiological agent has not been identified yet. It is believed to be an environmentally induced condition which predominantly affects males from paddy farming communities [1,6]. Furthermore, clustering of the disease around water reservoirs has been observed [3]. The common known causes of chronic kidney disease such as diabetes and essential hypertension are unlikely causes $[2,6]$. The cause is unlikely to be Ochrotoxin A, previously implicated in Balkan endemic nephropathy [7]. Some of the implicated aetiological factors that are under investigation include heavy metals, high ground water fluoride and agrochemicals [8-11].

Tubulointerstitial damage has been identified as the major pathological lesion in CKDU [12]. However, the exact pathogenesis is not well understood, as most tissue examinations have been performed in patients with advanced kidney disease when pathological changes are

Departments of ${ }^{1}$ Pathology and ${ }^{2}$ Microbiology Faculty of Medicine, University of Peradeniya, ${ }^{3}$ Nephrology and Transplant Unit, Teaching Hospital Kandy and ${ }^{4}$ Nephrology Unit, General Hospital Anuradhapura, Sri Lanka.

Correspondence: SW, e-mail: <suwijetunge@gmail.com>. Received 6 June 2013 and revised version accepted 12 September 2013. Competing interests: none declared. 
non specific. Therefore, we studied renal biopsies of asymptomatic individuals living in endemic regions, detected to have a kidney disease by screening programmes, to achieve the following objectives; 1) Describe the renal histopathology in kidney disease of unknown aetiology, detected by screening in those living in regions endemic for CKDU; 2) Correlate the histology with glomerular filtration rate and age; 3) Analyse the early pathological changes of endemic CKDU and determine the pathogenesis and progression of lesions.

\section{Methods}

This was a retrospective analysis of 211 renal biopsies, of asymptomatic individuals living in CKDU endemic regions, which were received by the Department of Pathology, Faculty of Medicine Peradeniya from 2007 to 2010. These renal biopsies had been performed for routine clinical management of the patients. Approval for the study was obtained from the Ethical Review Committee of the Faculty of Medicine, University of Peradeniya.

The target population was asymptomatic individuals living more than five years in areas identified as endemic for CKDU, and detected to have albuminuria using the dipstick method at field screening programmes. These individuals were referred to the nephrology clinics of regional hospitals and underwent routine diagnostic testing for renal diseases. Patients in whom renal biopsies were indicated, were referred to Teaching Hospital, Aunradhapura or Teaching Hospital, Kandy. Since this is a high risk population for chronic kidney disease and the pathogenesis and the natural history of the disease are not understood, the threshold for renal biopsy in CKDU endemic regions is lower than for the general population. Individuals with diabetes mellitus and long standing essential hypertension were not included in this category. Accordingly, indications for renal biopsy in this population were, positive albuminuria +1 or above on at least two consecutive dipstick tests and proteinuria more than 500 $\mathrm{mg} / 24$ hours, or proteinuria less than $500 \mathrm{mg} / 24$ hours with haematuria, or proteinuria less than $500 \mathrm{mg} / 24$ hours with renal insufficiency. Those who were in end stage renal disease were not biopsied.

Renal biopsies were collected in 10\% formaldehyde for routine processing and fresh for immunofluorescence. Formalin fixed paraffin embedded tissue sections were stained with haematoxylin and eosin, sliver stains for basement membranes and other special stains when required. Direct immunofluorescence for IgG, IgA, IgM and $\mathrm{C} 3$ was performed on fresh samples. Following the histological review, cases with identifiable glomerular disease, immunocomplex mediated disease and renal lesions secondary to systemic disease were excluded. Accordingly, from 234 biopsies, 211 (90.2\%) fulfilled the inclusion criteria and were selected as the study group.

The selected biopsies were reviewed for glomerular sclerosis (GS), interstitial fibrosis (IF), interstitial inflammation (II), tubular atrophy (TA) and hypertension associated changes in blood vessels. Absence or presence of these pathological features was recorded, and when present, graded on a scale from mild to severe. Since pathological changes were predominantly seen in the cortical regions, grading was performed based on the changes in the cortex. The criteria used to grade the severity of each pathological feature are as follows; small patches of interstitial fibrosis involving less than $10 \%$ mild fibrosis, multifocal fibrosis involving $10-50 \%$ moderate fibrosis and diffuse or coarse scarring involving more than $50 \%$ of the cortex severe fibrosis. Tubular atrophy was categorised as follows; presence of small groups of atrophic tubules involving less than $10 \%$ mild tubular atrophy multifocal tubular atrophy involving 10-50\% moderate tubular atrophy and widespread tubular atrophy involving more than $50 \%$ of the cortex severe tubular atrophy. Inflammation was categorised as follows; presence of small collections of lymphocytes and plasma cells involving less than $10 \%$ mild inflammation multifocal collections involving $10-50 \%$ moderate inflammation and large collections or diffuse infiltrations involving more than $50 \%$ of the cortex severe inflammation. Numbers of sclerosed glomeruli were given as a percentage of total number of glomeruli present in the biopsy. Hyaline arteriolosclerosis and intimal proliferations in medium size arteries were regarded as hypertension associated vascular changes, these features were not graded. Grading of the histological features was performed by two consultant histopathologists independently.

Based on the histological changes, observed cases were grouped into seven categories, from category 0 to category 6 using the following histological criteria.

- Category 0: No histological abnormalities

- Category 1: Interstitial fibrosis (IF) of any degree; absent or mild interstitial inflammation (II); tubular atrophy (TA) of any degree; normal glomeruli;

- Category 2: IF of any degree; absent or mild II; TA of any degree; glomerulur sclerosis (GS)

- Category 3: Moderate or severe IF and II (lymphocytes and/or plasma cell); TA of any degree; \pm GS

- Category 4: Absent IF; II and TA of any degree; $\pm \mathrm{GS}$

- Category 5: Severe II (lymphocytes and/or plasma cell) with widespread tubulitis warranting a diagnosis of interstitial nephritis; IF and TA of any degree; \pm GS

- Category 6: Severe IF and TA, II of any degree; GS - end stage kidney disease

Age and sex of the individuals and serum creatinine values at the time of biopsy were retrieved from the records. The glomerular filtration rate (GFR) was calculated using the modified diet in renal disease formula. 
Data was analysed using one way ANOVA test and the significance of the differences were indentified using Tukey's test. Association between GFR and histological categories was analysed calculating $\mathrm{R}^{2}$ value. The level of significance was set at 95\%. Categories 0, 4, 5 and 6 were excluded from the analysis due to small numbers.

\section{Results}

The age of the study group ranged from 10-65 years, the mean age was $36.8 \pm 14$ years. There were $153(72.5)$ males and $58(27.5 \%)$ females. The male to female ratio was 2.6. The study population consisted of 211 cases, 204 with histologically proven renal disease and 7 with normal histology. The distribution of cases according to histological categories 0 to 6 were, 7 (3.3\%), 71 (33.6\%), 53 (25.1\%), 63 (29.9\%), $0(0 \%), 2(0.9 \%)$ and $15(7.1 \%)$ respectively. All had interstitial fibrosis of varying degree and $86(42.2 \%)$ did not have any inflammation. In categories 1, 2, 3 and 6 the main tubular pathology was tubular atrophy and tubulitis was seen only focally. In category 5 , the degree of interstitial inflammation was greater than interstitial fibrosis and the main tubular change was wide spread tubulitis.

Severity of interstitial fibrosis, interstitial inflammation and tubular atrophy in each histological category is given in Table 1. In category 1, 62 (87.3\%) had interstitial fibrosis without interstitial inflammation; tubular atrophy when present was often mild or moderate. The rest (12.7\%) had mild interstitial inflammation in addition to fibrosis. Tubulitis was not present in any of the cases in category 1. In category 2, $24(45.3 \%)$ had interstitial fibrosis with or without tubular atrophy and the rest (54.7\%) had mild interstitial inflammation as well; the mean percentage glomerulosclerosis was $27.7 \%$. In category 3 , all had moderate to severe interstitial fibrosis and inflammation and the mean percentage glomerulosclerosis was $35.2 \%$. There were no cases in category 4 , (cases with interstitial inflammation not associated with interstitial fibrosis). In category 5, all cases had interstitial inflammation with interstitial fibrosis. However, unlike in other categories, interstitial inflammation with tubulitis was the predominant feature in this category. Category 6 represented advanced renal damage with glomerulosclerois, severe interstitial fibrosis and tubular atrophy and interstitial inflammation of varying degree. The mean percentage glomerulosclerosis was $77.7 \%$. Prevalence of hypertension associated vascular changes in each group was as follows; categories $0,1,2=0 ; 3=22(34.9 \%)$; category $4=0$, category $5=0$ and category $6=7(46.7 \%)$.

The mean age, serum creatinine levels, GFR and glomerulosclerosis percentage of each histological category are given in Table 2. Tukey's test was used to identify significant deference between categories. The mean age of histological category 1 was significantly lower than those of categories 2 and 3; category 1 vs 2 (95\% CI 11.11-20.97) and category 1 vs 3 (95\% CI 12.32- 21.72). The mean age of category 2 was not significantly different from category 3; (95\% CI -4.08-6.04). The mean GFR's of histological categories 1 to 3 were significantly lower than the preceding category; category 1 vs 2 (95\% CI -53.26 19.72 and category 1 vs $3(95 \%$ CI $-84.33,52.35)$ and category 2 vs 3 are (95\% CI -49.07-14.63). The mean GFR has declined from category 1 to 3 in a linear manner $\left(\mathrm{R}^{2}=0.998\right)($ Figure 1).

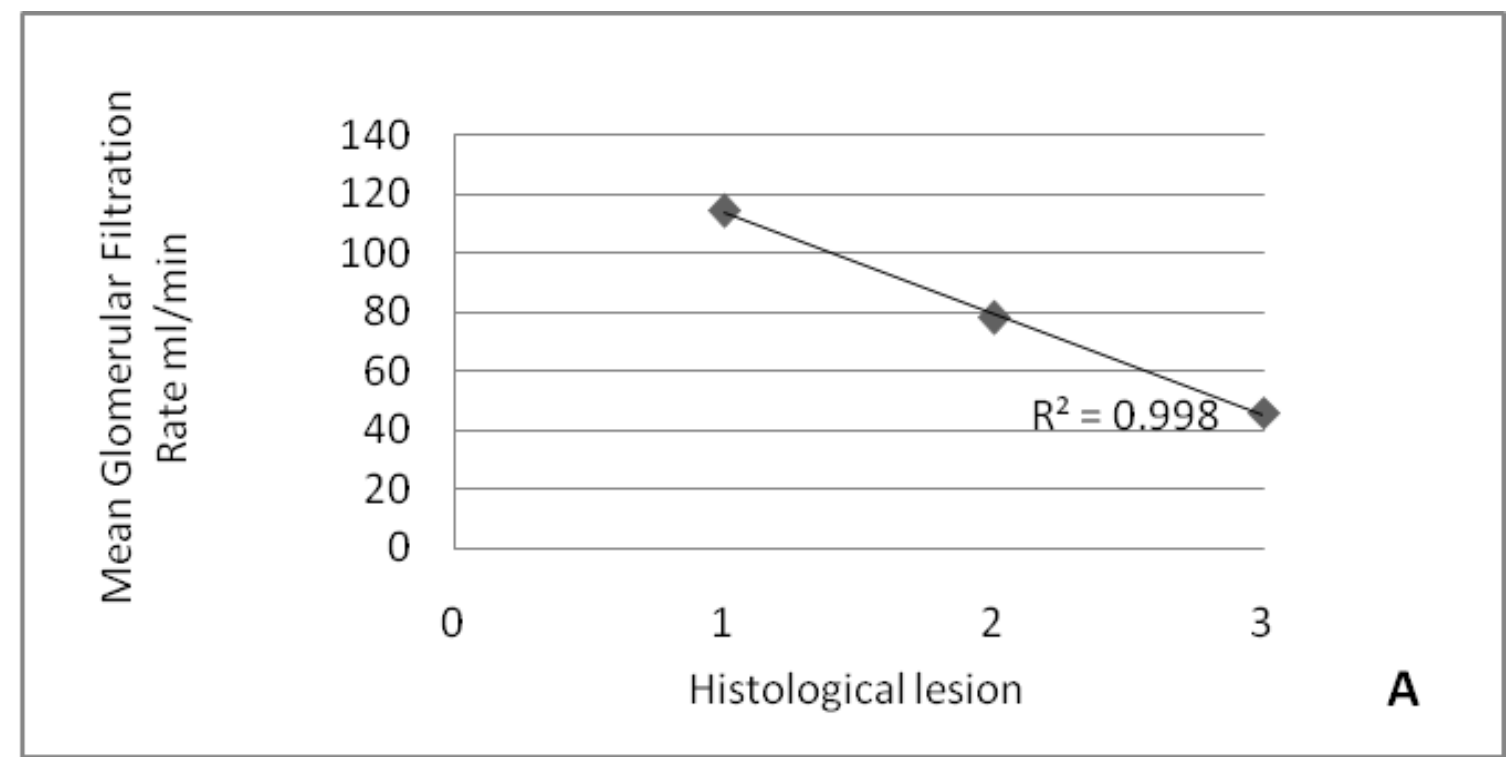

Figure 1. Change in mean glomerular filtration rate with renal lesions, categories 1 to 3. Figure shows that from renal lesions category 1 to 3 the mean glomerular filtration rate has declined in a linear manner. 


\section{Discussion}

In the study population, $88.6 \%$ of the cases belonged to categories 1, 2 and 3 and the main pathological features of were interstitial fibrosis, interstitial inflammation and tubular atrophy of varying degree. Widespread tubilitis was not a feature in these categories. These changes are non specific and do not signify an aetiology agent. The predominant pathological change in the study sample was interstitial fibrosis; in $42.2 \%$ the main pathological change was interstitial fibrosis but these cases did not have any inflammation. Furthermore, there were no cases with interstitial inflammation without interstitial fibrosis (category 4). According to renal functions, as indicated by mean GFR, it appears that, in this study population, the earliest pathological manifestation is category 1 which has normal GFR with histologic evidence of renal injury (Table 2). In category $1,87.3 \%$ had interstitial fibrosis without inflammation and tubular atrophy when present was usually mild (Table 1). Therefore, interstitial fibrosis appears to be the earliest detectable pathological change in this asymptomatic population.

\section{Table 1. Severity of histological changes (interstitial fibrosis, interstitial inflammation and tubular atrophy) in histological categories 1 to 6. Severity of each histological change is graded on a scale mild to severe (criteria described in methodology)}

\begin{tabular}{|c|c|c|c|c|c|c|c|c|c|c|c|c|c|}
\hline \multirow{2}{*}{$\begin{array}{l}\text { Histological } \\
\text { category }\end{array}$} & \multirow{2}{*}{$\begin{array}{c}\text { Total } \\
\text { Number } \\
\text { of cases }\end{array}$} & \multicolumn{4}{|c|}{ Interstitial fibrosis } & \multicolumn{4}{|c|}{ Interstitial inflammation } & \multicolumn{4}{|c|}{ Tubular atrophy } \\
\hline & & $\mathrm{No}$ & mild & $\bmod$ & severe & No & mild & $\bmod$ & severe & No & mild & $\bmod$ & severe \\
\hline 1 & 71 & 0 & 58 & 12 & 1 & 62 & 9 & 0 & 0 & 28 & 38 & 4 & 1 \\
\hline 2 & 53 & 0 & 15 & 35 & 3 & 24 & 29 & 0 & 0 & 7 & 14 & 31 & 1 \\
\hline 3 & 63 & 0 & 0 & 31 & 32 & 0 & 0 & 45 & 18 & 0 & 3 & 37 & 23 \\
\hline 4 & 0 & 0 & 0 & 0 & 0 & 0 & 0 & 0 & 0 & 0 & 0 & 0 & 0 \\
\hline 5 & 2 & 0 & 0 & 2 & 0 & 0 & 0 & 0 & 2 & 0 & 0 & $28 *$ & 0 \\
\hline 6 & 15 & 0 & 0 & 0 & 15 & 0 & 1 & 0 & 14 & 0 & 0 & 4 & 11 \\
\hline
\end{tabular}

* there was widespread tubulitis, in other lesions tubulitis was either absent or when present only focal

Table 2. Mean age, mean GFR and mean serum creatinine level in each histological category Abbreviations, SE, standard error of the mean, GFR, glomerular filtration rate

\begin{tabular}{|c|c|c|c|c|c|}
\hline $\begin{array}{c}\text { Histological } \\
\text { category }\end{array}$ & $\begin{array}{l}\text { Total number } \\
\text { of cases } \\
\quad(S E)\end{array}$ & $\begin{array}{c}\text { Mean age } \\
\text { (years) } \\
(S E)\end{array}$ & $\begin{array}{l}\text { Mean GFR } \\
\text { (ml/minute) }\end{array}$ & $\begin{array}{c}\text { Mean serum } \\
\text { creatinine mol/l) (SE) } \\
\text { (normal range: } \\
53-115)\end{array}$ & $\begin{array}{c}\text { Mean \% sclerosed } \\
\text { glomeruli* } \\
(S E)\end{array}$ \\
\hline 0 & $7(3.3 \%)$ & $28.0(5.5)$ & $108.2(512)$ & $91.8(2.9)$ & 0 \\
\hline 1 & $71(33.6 \%)$ & $26.3(1.5)$ & $114.4(5.3)$ & $64.8(5.02)$ & $0(0)$ \\
\hline 2 & $53(25.1 \%)$ & $42.3(1.6)$ & $77.9(6.3)$ & $103.2(8.49)$ & $27.7(1.9)$ \\
\hline 3 & $63(29.9 \%)$ & $43.3(1.3)$ & $46.1(2.7)$ & $135.8(9.93)$ & $35.2(3)$ \\
\hline 4 & $0(0 \%)$ & $0(0)$ & $0(0)$ & $0(0)$ & $0(0)$ \\
\hline 5 & $2(0.9 \%)$ & $25(12.1)$ & $53.1(17.2)$ & $158.8(27.07)$ & $0(0)$ \\
\hline 6 & $15(7.1 \%)$ & $42.8(3.4)$ & $34.4(3)$ & $175.6(19.15)$ & $77.5(3.1)$ \\
\hline
\end{tabular}

*percentage sclerosed glomeruli is given as the number of sclerosed gomeruli as a percentage of total glomeruli in the biopsy. 
There are two possible explanations for this; interstitial fibrosis in these cases represent healed foci of tubulo-interstitial inflammation or the initial interstitial response to the injury is fibrosis. There is evidence to suggest that tubular epithelial damage occurs early in Sri Lankan endemic CKDU as detected by increased excretion of urinary $\alpha_{1}$ - microglobulin in the early stages of the disease [13]. If the initial tubular injury results in tubular epithelial cell necrosis, as in exposure to higher concentrations of a toxin, the more likely interstitial response is tubulo-interstitial inflammation characterized by tubilitis and interstitial infiltration of lymphocytes and plasma cells. It is possible that during the asymptomatic period patients are free of such active inflammation. Whereas, in tubular epithelial dysfunction, as in chronic low grade exposure to a toxin, the more likely initial outcome is interstitial fibrosis. The implicated mechanisms by which non lethal tubular epithelial injury lead to interstitial fibrosis are G2/M cell cycle arrest, cellular apoptosis and epithelial mesenchymal transition [14-16). Therefore, it is possible that the interstitial changes in Sri Lankan endemic CKDU could be due to either episodes of acute tubulo-interstitial inflammation followed by healing by fibrosis, giving rise to progressive interstitial fibrosis caused by episodic exposure to higher concentrations of toxin or progressive interstitial fibrosis from the onset due to chronic low grade exposure to toxin or a combination of both.

Furthermore, since the pathological changes were predominantly seen in the cortical region, it is possible that the tubular damage is mostly in the proximal convoluted tubules. More studies are needed to confirm this.

Regardless of the initial response, once established, interstitial fibrosis plays a pivotal role in the progression of renal diseases producing a vicious cycle of tubulointerstitial and ultimately glomerular changes. Rarefaction of peritubular microvasculature and reduction in oxygen diffusion are effects of interstitial fibrosis which give rise to ischaemic tubular epithelial injury and tubular atrophy [17-18]. In such circumstances interstitial inflammation could be a secondary response to ischaemic tubular epithelial cell necrosis. Interstitial inflammation itself induces further interstitial fibrosis. Tubular atrophy and fibrosis induced peritubular capillary obliteration induce glomerular ischaemia and increase in intraglomerular pressure ultimately resulting in glomerular sclerosis, which in turn produces further interstitial fibrosis due to ischemia secondary to reduced perfusion by efferent arterioles.

The two cases in category 5 had active, severe, chronic interstitial nephritis (Table 1). These two cases could represent either an active phase of endemic CKDU or non endemic chronic interstitial nephritis. Category 0 may represent sampling errors. The number of cases in category 6 may not be correctly represented here because these patients usually have shrunken kidneys on imaging and are in end stage renal disease, and were not biopsied.

In this sample, GFR declined from category 2 onwards, which coincides with the appearance of glomerulosclerosis. In category 3, with established interstitial fibrosis, interstitial inflammation, tubular atrophy and glomerulosclerosis, GFR had further declined (Figure 1). In category 3 there were only 4 cases without sclerosed glomeruli and this could be due to sampling error. Reduction of GFR had a linear correlation from categories 1 to 3. i.e., $\mathrm{R}^{2}$ value 0.998 (Figure 1 ).

The mean age of patients with early disease (category 1) was 26.3 years, which suggests that the exposure occurs relatively early in the lives of those affected. In the more advanced cases (categories 2 and 3) the mean ages (42.3 and 43.3 years respectively) were significantly higher which shows the slow progression of the disease.

Absence of hypertension associated vascular changes in the early stages of the disease makes hypertension an unlikely aetiological agent. In late cases, hypertension is probably secondary to advanced renal damage.

In conclusion, it appears that early disease among asymptomatic patients is characterised by interstitial fibrosis without significant interstitial inflammation and glomerular sclerosis and preserved glomerular function. A role for interstitial inflammation in the initiation of the disease is not apparent. However, interstitial inflammation appears to have a definite role in the progression of the disease.

\section{Limitations of the study}

Since this was a retrospective study performed on clinical samples there were several limitations. The study sample consisted of a carefully selected group of participants who were eligible to undergo renal biopsies, not a random group and therefore, is likely to be biased. Due to ethical issues it is difficult to perform renal biopsies on randomly selected participants. Furthermore, due to lack of diagnostic criteria, case definition used for CKDU relied almost entirely on residence in an endemic area. Since this is a retrospective laboratory study, clinical details obtained were limited to what was available in the clinical information form. However, this study provides baseline information on histological changes of the renal diseases prevalent in the endemic regions. These results may be helpful in designing studies to elucidate the nature of the disease and formulating diagnostic criteria.

\section{Acknowledgements}

Authors acknowledge the technical assistance of Mr. G. H. D. S. Chandraprabha and Mrs. H. M. N. D. Herath. 


\section{References}

1. Athuraliya TNC, Abeysekera DTDJ, Amerasinghe PH, Kumarasiri PVR, Dissanayake V. Prevalence of chronic kidney disease in two tertiary care hospitals: high proportion of cases with uncertain aetiology. Ceylon Medical Journal 2009; 54: 23-5.

2. Athuraliya NT, Abeysekera TD, Amerasinghe $\mathrm{PH}$, Kumarasiri R, Bandara P, Karunaratne U, Milton AH, Jones AL. Uncertain etiologies of proteinuric-chronic kidney disease in rural Sri Lanka. Kidney International 2011; 80: 1212-21.

3. Jayasekara JMKB, Dissanayaka DM, Adhikari SB, Bandara P. Geographic distribution of chronic kidney disease of unknown aetiology in North Central region of Sri Lanka. Ceylon Medical Journal 2013; 58: 6-10.

4. Chandrajith R, Nanayakkara S, Itai $\mathrm{K}$, et al. Chronic kidney diseases of uncertain etiology (CKDUe) in Sri Lanka: geographic distribution and environmental implications. Environmental and Geochemical Health 2011; 33: 267-78.

5. World Health Organization report on chronic kidney disease of unknown aetiology in Sri Lanka. http://www. lankaweb. com/news/items/2012/08/15/world-health-organizationwho-report-on-chronic-kidney-disease-of-unknownetiology-ckdu-in-sri-lanka/. Accessed on 10.04.2013.

6. Wanigasuriya KP, Peiris-John RJ, Wickremasinghe R, Hittarage A. Chronic renal failure in North Central Province of Sri Lanka: an environmentally induced disease. Transactions of Royal Society of Tropical Medicine and Hygiene 2007; 101: 1013-7.

7. Wanigasuriya KP, Peiris H, Ileperuma N. Could ochratoxin A in food commodities be the cause of chronic kidney disease in Sri Lanka? Transactions of the Royal Society of Tropical Medicine and Hygiene 2008; 102: 726-8.

8. Illeperuma OA, Dharmagunawardhane HA, Herarh KPRP. Dissolution of aluminum from substandard utensils under high fluoride stress: A possible risk factors for chronic renal failures in the North-Central Province. Journal of the National Science Foundation of Sri Lanka 2009; 37: 219-22.

9. Peiris-John RJ, Wanigasuriya JKP, Wickremasinghe AR, Dissanayaka WP, Hittarage A. Exposure to acetylcholine- sterase-inhibiting pesticides and chronic renal failure. Ceylon Medical Journal 2006; 51: 42-3.

10. Wanigasuriya KP, Peiris-John RJ, Wickremasinghe R. Chronic kidney disease of unknown aetiology in Sri Lanka: is cadmium a likely cause? BMC Nephrol. 2011; 12: 32.

11. Bandara JMPS, Senevirathna DMAN, Dasanayake DMRSB, et al. Chronic Renal Failure among farm families in cascade irrigation system in Sri Lanka associated with elevated dietary cadmium levels in rice and freshwater fish (Tilapia). Environmental Geochemistry and Health 2008; 3: $465-78$.

12. Nanayakkara S, Komiya T, Ratnatunga N, et al. Tubulointerstitial damage as the major pathological lesion in endemic chronic kidney disease among farmers in North Central Province of Sri Lanka. Environmental Health and Preventive Medicine 2012; 17: 213-21.

13. Nanayakkara S, Senevirathna ST, Karunaratne U, et al. Evidence of tubular damage in the very early stage of chronic kidney disease of uncertain etiology in the North Central Province of Sri Lanka: a cross-sectional study. Environmental Health and Preventive Medicine 2011; 17: 109-17.

14. Yang L, Besschetnova TY, Brooks CR, Shah JV, Bonventre JV. Epithelial cell cycle arrest in G2/M mediates kidney fibrosis after injury. Nature Medicine 2010; 16: 535-43.

15. Thomas GL, Yang B, Wagner BE, Savill J, Nahas AMEI. Cellular apoptosis and proliferation in experimental renal fibrosis. Nephrology Dialysis Transplantation 1998; 13: 2216-26.

16. Iwano M, Plieth D, Danoff TM, Xue C, Okada H, Neilson EG. Evidence that fibroblasts derive from epithelium during tissue fibrosis. Journal of Clinical Investigations 2002; 110: 341-50.

17. Ohashi R, Kitamura H, Yamanaka N. Peritubular capillary injury during the progression of experimental glomerulonephritis in rats. Journal of the American Society of Nephrology 2000; 11: 47-56.

18. Bohle A, Mackensen-Haen S, Wehrmann M. Significance of post-glomerular capillaries in the pathogenesis of chronic renal failure. Kidney and Blood Pressure Research 1996; 19: 191-5. 